\title{
DEL CAMBIO CONCEPTUAL A LA ADQUISICIÓN DE CONOCIMIENTOS: Algunas reflexiones sobre las concepciones alternativas y el cambio conceptual de J.M. Oliva
}

\author{
MARÍN MARTÍNEZ, NICOLÁS \\ E-mail: nmarin@ualm.es
}

\begin{abstract}
Aprovecho la invitación que me brinda Enseñanza de las Ciencias para colocarme al otro lado del espejo, allí donde expresarme de un modo espontáneo y flexible para sembrar cierta «polémica razonable» que sirva de estímulo para la reflexión y la discusión sobre este tema (hasta ahora nadie se anima a tomar el reto del debate, se quejan en la revista). Algo semejante me ocurre cuando en la zona donde se concluyen los artículos busco el debate insertando argumentos que inviten a ello.
\end{abstract}

De lo que no se puede dudar es del espíritu emprendedor, tenaz y casi temerario de los que pululamos por el ámbito de la didáctica de las ciencias (DC). Tomando como ejemplo la comunidad hispana, se percibe que la gran mayoría de sus integrantes poseen una formación universitaria inicial en disciplinas de ciencias, que después se ha ido completando a título personal, husmeando por cualquier área de conocimiento que pueda resolver los problemas encontrados en la enseñanza y aprendizaje de las ciencias (Gutiérrez, 1987, 1990).

Con la persistencia de termitas, no se escatiman esfuerzos por entrar en discusiones de filosofía de las ciencias con Kuhn, Popper, Toulmin, Lakatos, Bunge..., por recorrer la historia de la ciencia junto a Koyre, Crombie, Meyerson..., por entrar en las polémicas más crudas sobre aprendizaje y, por si no fuera suficiente, compitiendo con Piaget, Ausubel, Rumelhart, Vigotsky..., proponiendo nuevos modelos de organización del conocimiento y aprendizaje (Pozo, 1989; Gutiérrez, 1992; Luffiego et al., 1994; Marín, 1994; Posada, 1996). Des- graciadamente no se puede decir que poseamos aún la sorprendente y eficaz coordinación y organización de las termitas que elevan estructuras arquitectónicas para resolver sus problemas de ventilación.

En el edificio de la DC se perciben partes, como las que se apoyan en la historia y filosofía de las ciencias (HFC), más adelantadas que otras; en realidad eso ha ocurrido porque ahí trabajan la mayor parte de los obreros -la mayoría de trabajos publicados tienen como principal referente la $H F C$-, tienen los ladrillos más cerca -la HFC es lo más cercano a la formación científica inicialy los promotores les liberan las mayores partidas presupuestarias -los proyectos y trabajos fundamentados en la HFC, por ahora, son los más subvencionados.

Cuando algunos capataces hablan de ir colocando la bandera del último piso -al afirmar que la DC es un cuerpo de conocimientos coherente y suficientemente consensuado-, los sufridos obreros de la parte atrasada del edificio -más formados en los pasajes representacionales de la cognición del alumno- se quejan y polemizan sobre deficiencias en la construcción.

El problema se agrava cuando parece no estar muy clara la forma final que debe adoptar el edificio de la DC, qué pilares y estructuras tendrían que soportarlo. Incluso se discute si los pilares son necesarios (Marín, Solano y Jiménez Gómez, en prensa).

Quizá por esto, en los últimos años, desde las editoriales de las revistas más relevantes del ámbito se sugiere en 
qué zonas del edificio se debería trabajar más, se esbozan modos de levantar paredes más sólidas, se indican qué paredes se deberían echar abajo y se comienza a establecer criterios más precisos para contratar obreros mejor cualificados (Duschl, 1994; Kyle, 1994; Gilbert, 1995; Sanmartí y Azcárate, 1997). Aun así, creo que nos queda mucho por hacer para que el edificio sea tan funcional como el de las termitas.

\section{DEBATES HISPANOS SOBRE EL CAMBIO CONCEPTUAL}

José María Oliva realiza, salpicada de excelentes intuiciones, una bien documentada y estructurada revisión crítica de la propuesta de cambio conceptual. Lo que más me ha sorprendido es que, desde otra perspectiva y con otro modo de abordar los datos bibliográficos, presenta notables coincidencias con mis argumentos críticos; concretamente, ambos señalamos que la propuesta de cambio conceptual:

a) no contempla los cambios procesuales ni los referidos a aspectos generales de la cognición, relevantes para las adquisiciones conceptuales;

b) sugiere un modo de aprendizaje basado en la simple permuta de ideas, que es bastante cuestionable;

c) posee un campo de aplicación restrictivo y su validez es discutible;

d) carece de una visión analítica del proceso de adquisición de nuevos conocimientos.

$\mathrm{El}$ aspecto crítico defendido por Oliva-existe más de un mecanismo de cambio conceptual- es planteado en mi trabajo de un modo diferente. Así, en el plano de la enseñanza se afirma que existen otras estrategias de enseñanza [diferentes al cambio conceptual] más adecuadas para tratar los distintos tipos de limitaciones cognoscitivas encontradas en el alumnado y en el plano del aprendizaje existen diferentes mecanismos de adquisición y desarrollo cognoscitivo y ninguno se parece al sugerido por el cambio conceptual; el único que presenta cierta analogía es el de asimilación seguido de un proceso [largo] de diferenciación progresiva.

Y es que la fuerte difusión de la propuesta de cambio conceptual facilita caer en el tópico usual de considerarlo como sinónimo de aprendizaje; de ahí se sigue que tipos de cambio conceptual sean usados como sinónimos de tipos de aprendizaje. Esta confusión «encorseta» y erosiona nuestro ámbito, pues invita a fundamentar toda una investigación de enseñanza y aprendizaje en «la teoría del cambio conceptual» o en «la visión constructivista del cambio conceptual» cuando éste, sin entrar a discutir su validez, no va más allá de ser una orientación didáctica de las muchas que existen o pueden existir en nuestro ámbito (Marín, 1998).

La interesante sugerencia final de Oliva de asumir $e$ incorporar de alguna forma los presupuestos teóricos revisados, supongo que no será para llevarla a cabo sumando los diversos planteamientos teóricos que revisa, algunos dispares entre sí. Habría que recordar la advertencia de Coll (1990) en la que, aceptando como conveniente realizar aportaciones de las diversas teorías de aprendizaje al plano de la enseñanza, éstas se deberían hacer con precaución dado que existe el riesgo de desvirtuar las propuestas originales, provocar contradicciones o incoherencias, etc. El peligro de hacer mezclas de teorías es tanto mayor si éstas poseen fundamentos tan dispares como son las epistemologías científicas e individuales.

La distinción entre ambos tipos de epistemologías es la base de la crítica teórica que realizo del cambio conceptual, y es que la formación del conocimiento científico tiene connotaciones bien diferentes al cotidiano o individual y las teorías que dan cuenta de uno u otro también son necesariamente diferentes.

Por ejemplo, percibo como peligrosos los paralelismos que traza Oliva entre la asimilación piagetiana y los procesos de captura, crecimiento, ajuste o reestructuración débil y entre la acomodación y la reestructuración fuerte, entre otras razones porque los supuestos básicos que usan los constructos citados son bien distintos y porque Piaget consideraba, estuviera más o menos equivocado, los procesos de asimilación y acomodación como las dos caras de la moneda de la adaptación (Bringuier, 1977).

\section{La sorprendente mezcla armoniosa del cambio conceptual de Ignacio Pozo}

Ignacio Pozo (1989), como José María Oliva, realiza alusiones a las reestructuraciones débiles y fuertes de Carey (1991), en este caso como preámbulo a su propuesta de cambio conceptual. Después evoca la teoría de Lakatos para relacionar los cambios en el cinturón protector, con la reestructuración débil, y los cambios del núcleo duro de la teoría, con la reestructuración fuerte, y en este punto, introduce las conocidas condiciones del cambio conceptual como la propuesta idónea para cambiar las concepciones espontáneas. (Siempre he creído que Pozo ha dado demasiada credibilidad a las propuestas del constructivismo social) (Marín et al., en prensa.) Finalmente realiza su propuesta de cambio conceptual.

Así, después del excelente repaso de las distintas teorías sobre el aprendizaje -el modo de usar los comentarios críticos para trazar la secuencia de presentación de teorías, lo cual me parece el mejor logro de su revisión-, Ignacio Pozo remata su recorrido con un modelo que denomina, para mi sorpresa, cambio conceptual, que resulta ser un perfecto ejemplo de mezcla de retazos de teorías.

El modelo de «cambio conceptual» de Pozo orquesta, en un difícil equilibrio, los aprendizajes por acumulación con los de reestructuración, los procesos de toma de conciencia de Piaget (1976) y otros más cercanos a la noción de darse cuenta y caer en la cuenta de Claxton 
(1984), el aprendizaje por instrucción con otros tipos, las conductas alfa, beta y gamma de la teoría de equilibración de Piaget (1978) con las reestructuraciones fuertes y débiles de Carey (1991).

En mi opinión, el modelo de Pozo ofrece una solución coherente a los problemas de formación y asignación de significados de las teorías del aprendizaje mecanicistas y, al integrar la acumulación con la reestructuración, da salida a la paradoja del aprendizaje de las teorías organicistas. Si Oliva reclama una visión más analítica sobre los mecanismos de aprendizaje (él dice de cambio conceptual), creo que ahí la tiene.

Ahora bien, admitiendo la bondad del modelo de Pozo, cabe preguntarse de qué teoría sobre la formación y organización del conocimiento se deduce. El modelo toma prestados elementos de varias teorías y construye un todo que parece armonioso, pero con las teorías no podemos hacer lo mismo. En esto la DC necesitaría de la psicología cognitiva una solución que ahora no parece aportar su diversidad y dispersión (irónico sería que más pronto que tarde, cual termitas, la propongamos desde la DC).

Percibo en el modelo de Pozo algo que chirría, y es la indiferenciación entre los términos concepto y esquema, que por las razones dadas en mi crítica al cambio conceptual no son sinónimos (por ejemplo, se me hace difícil asimilar la frase la integración de esquemas supone una considerable economía cognitiva, ya que reduce el número de conceptos que forman parte del núcleo central de la teoría). Además, el uso funcional que hace de esquema unas veces se aproxima al de la teoría de Piaget (1978) y otras, a la visión de Rumelhart y Ortony (1982) cuando tienen connotaciones y contextos diferentes. Opino que el modelo de Pozo no se debería llamar de cambio conceptual sino de aprendizaje y desarrollo de esquemas.

\section{El complemento metodológico que Daniel Gil y sus colaboradores ponen al cambio conceptual}

Además de gran comunicador, cabe destacar el importante papel de Daniel Gil como difusor de los avances anglosajones en la incipiente comunidad hispana de DC de comienzos de los ochenta; en particular habría que destacar la fuerte incidencia que han tenido sus propuestas de cambio metodológico y resolución de problemas en el profesorado de enseñanzas medias y en el desarrollo curricular de éstas en contextos oficiales, sin olvidar sus importantes aportaciones en otras direcciones (Gil, 1991).

Él supo retomar la metodología científica para elevarla a propuesta didáctica coherente hasta el punto de desafiar la hegemonía del cambio conceptual. Al igual que Piaget cambiaba la forma a la plastilina para identificar los niños no conservadores de la cantidad, Daniel Gil supo presentarnos dicha metodología de forma reiterada y dándole variadas formas. Además, supo revestirla del fundamento bibliográfico necesario para sacarla del uso «doméstico» que le dábamos en el aula y pasearla por las revistas más relevantes del ámbito. Mientras observábamos con cierto escepticismo cruzar fugaces las «propuestas de cambio» por el firmamento didáctico, algunos continuábamos enfrascados en una lenta formación sobre aprendizaje de las ciencias.

Los buenos resultados de aula del cambio metodológico habría que verlos como excesivamente optimistas cuando el test de Shayer (1979) indica que no más del 30\% del alumnado hispano llega a abordar los problemas con planteamientos hipotético-deductivos y presentar cierta capacidad para controlar las variables del problema (Marín, 1986). En otra serie de investigaciones (Marín, 1984a; Marín, 1984b; Marín, 1985) se mostraba la dificultad de futuros maestros para hacer las prácticas de física mediante procedimientos hipotéticos-deductivos a partir del planteamiento de un problema; sin embargo, los resultados eran claramente superiores cuando éste mismo era abordado con una estrategia inductiva de descubrimiento dirigido con fundamento piagetiano.

Sin embargo, los resultados que hablan de la bondad del aprendizaje por descubrimiento piagetiano quedan puestos en entredicho por Gil (1993), que argumenta que dicho método tiene poco que ver con la visión actual de lo que constituye el trabajo científico. Sin embargo, el mismo autor continúa diciendo que la propuesta de enseñanza expositiva de Ausubel sí que supone un progreso, pues está más acorde con la naturaleza de la ciencia, y señala que una revisión atenta de la propuesta de Ausubel muestra una indudable coherencia con las tesis básicas de la epistemología de la ciencia, si bien fracasa al no considerar un tiempo propio para los alumnos. Este concluye: el esfuerzo de fundamentación habría que dirigirlo a la atención de las analogías entre la actividad de los científicos y la de los alumnos.

La cuestión es: ¿Se pueden evaluar teorías de aprendizaje, o sus consecuencias didácticas tomando criterios de las teorías sobre epistemología de la ciencia?

Aunque percibo el cambio metodológico como una propuesta que complementa, incluso supera, al cambio conceptual (Martínez Torregrosa et al., 1993), no creo que pueda ser eficaz mientras el docente no tenga cierta información sobre las capacidades y limitaciones cognoscitivas del alumnado acerca de los procedimientos científicos que se desean poner en juego en la enseñanza. $\mathrm{Y}$ aún disponiendo de esta información, creo que la epistemología de la ciencia no puede ser el gran pilar que fundamenta la DC, este mérito debería compartirlo con los distintos tipos de mecanismos cognoscitivos que dan cuenta del aprendizaje de los diversos contenidos de ciencias, porque, se enseñe como se enseñe, siempre está ahí el alumno que debe aprender.

Y es que la analogía del alumno como científico novel, -y sus repercusiones didácticas- está bien en una primera aproximación, pero sería bueno buscar ayuda en los detalles que ofrecen los mecanismos de adquisición cognoscitiva para responder con eficacia a las situaciones problemáticas de enseñanza y aprendizaje poniendo 
los puentes necesarios entre el conocimiento de uno y otro (Marín, 1998).

\section{REFLEXIONES Y OPINIONES A MODO DE CONCLUSION}

Junto a la copiosa invasión de todo tipo de productos lúdicos, culturales, tecnológicos, etc., a nuestro ámbito también llegaron de la otra parte del Atlántico los mapas conceptuales, el aprendizaje significativo, los misconceptions, el cambio conceptual, etc.

En mi opinión, los citados «productos didácticos» están sobrevalorados, ya que han sido objeto de una gran difusión -las revistas más relevantes de nuestro ámbito son americanas- y tienen en común el ser propuestas didácticas sencillas «cercanas» a los conocimientos de nuestra formación universitaria. Así:

- La propuesta de usar como técnica didáctica los mapas conceptuales no va más allá y no supone nada nuevo respecto a los organigramas y esquemas gráficos que ya se hacían para estructurar y secuenciar los contenidos académicos; sin embargo, bajo el bonito término de mapa conceptual, la técnica ha tenido una gran aceptación entre docentes e investigadores, quizá porque las instrucciones que se dan para su diseño no requieren de una formación especial o adicional (Novak y Gowin, 1988). Como método para el diagnóstico del conocimiento del alumno, en la medida que se enfatiza el significante sobre el significado, resulta ser bastante pobre sobre otras técnicas, y se corre el peligro de tomar una información bastante sesgada (Marín, 1995).

- Cuando se percibe que los «errores conceptuales» de comienzos de la década de los ochenta recogen del alumno, sobre todo, su desconocimiento del tema, se emigra lentamente hacia los «esquemas alternativos» al seleccionar aquellas respuestas que presentan mayor estabilidad y consistencia (Oliva, 1996), pero al seguir manteniendo los mismos defectos metodológicos (Marín, Jiménez Gómez y Solano, 1996) hace que la progresión en esta línea de investigación sea nimia (Jiménez Gómez, Solano y Marín, 1997).

- La propuesta de aprendizaje verbal significativo de Ausubel basada en el intuitivo y sencillo mecanismo de ligar o relacionar los nuevos materiales con las ideas que ya posee el alumno puede ser útil cuando los objetivos de enseñanza de las ciencias supongan comprensión de información verbal de conocimientos declarativos (Lawson, 1983; Aliberas et al., 1989; Beltrán, 1993; García Madruga, 1995), siempre que el alumno tenga cierto desarrollo cognoscitivo que incluya algún dominio de las relaciones de inclusión y de estrategias hipotético-deductivas, y el docente haya podido conformar un organizador previo acorde con lo anterior y con la estructura cognoscitiva del alumno, tarea poco sencilla (Pozo, 1989; García Madruga, 1995). Si no son suficientes estas limitaciones, Claxton (1984), por un lado, muestra la existencia de otras estrategias de comprensión verbal diferentes a las de Ausubel y, por otro, los objetivos de enseñanza de la ciencia más relevantes, como la adquisición de contenidos procesuales y la transferencia de conocimientos, quedan sin respuesta (Aliberas et al., 1989; Beltrán, 1993).

- Sobre las limitaciones del cambio conceptual creo que ya se ha hablado suficiente entre la revisión crítica de Oliva y la mía.

Si estas propuestas didácticas no son tan buenas como pudiera sugerir su fuerte difusión, ¿qué alternativa se debería tomar? ¿Existen otras alternativas? Nuevamente voy a retomar la revisión de Ignacio Pozo (1989) entre las distintas teorías sobre aprendizaje -excelente para una rápida actualización de los que trabajamos en DCen el punto donde deja entrever las notables diferencias entre norteamericanos y europeos para plantear y abordar el tema-los primeros con planteamientos más mecanicistas y los segundos más organicistas.

Creo que no se pueden extrapolar estas diferencias al ámbito de DC, entre otras razones, porque el desarrollo hispano de este cuerpo de conocimiento siempre ha ido a remolque de los productos exportados, pero ¿existe la posibilidad de tomar la iniciativa didáctica?

No estoy sugiriendo un rechazo sólo porque el producto venga del oeste, ni tampoco tener permanentemente abiertos los brazos para acoger cualquier iniciativa nuestra. Más bien invito a fomentar la formación propia para elevar el sentido crítico ante productos didácticos importados de fuerte difusión y, si es posible, anteponer iniciativas ante el fácil recurso de aplicar las recetas que nos llegan.

Frente al desenfrenado consumo de productos americanos, personalmente prefiero alinearme a la corriente cultural europea que pretende fomentar nuestra producción y con ello nuestra identidad. Por eso y por su mayor calidad, prefiero anteponer las entrevistas individuales piagetianas, y su meticuloso modo de hacer, a la deficiente metodología del «movimiento de las concepciones alternativas» (Jiménez Gómez, Solano y Marín, 1997), a las ricas propuestas sobre la toma de conciencia, a la equilibración de las estructuras cognoscitivas y los procesos de asimilación y acomodación a las discutibles «asociaciones o enganches» del aprendizaje significativo o a la molar propuesta de cambio conceptual.

En cuanto a posibles aportaciones hispanas, estoy convencido de que el modelo de aprendizaje de Pozo aportaría un soporte sólido para llevar a cabo investigaciones de enseñanza y aprendizaje de contenidos de ciencias, que la propuesta de Gil y sus colaboradores, con los matices antes apuntados, es la vía eficaz para fomentar el conocimiento procesual e hipotético-deductivo de los alumnos, y que no hay que olvidar las notables y bien fundamentadas aportaciones didácticas que continuamente realizan nuestros compañeros latinoamericanos (Villani, Pacca, Saraiva, Niaz, Sebastiá, etc.). 
Bueno sería quitar el polvo a los libros de Piaget que teníamos confinados en un rincón de nuestra estantería y asimilar lo mucho de bueno que tienen, obviando lo que las investigaciones actuales han mostrado más débil de su teoría. Creo que el mayor pecado de Piaget residió en no saber vender sus productos, con ese estilo poco didáctico que tenía para expresar sus ideas (ni un mal dibujo que ilustre alguno de sus famosos experimentos). Aún así, opino, con Vuyk (1985), que merece la pena retomarlo una vez más; todavía quedan en su prolífera bibliografía muchas experiencias y muchas ideas que podrían enriquecer nuestro dominio.

\section{REFERENCIAS BIBLIOGRÁFICAS}

ALIBERAS, J., GUTIÉRREZ, R. e IZQUIERDO, M. (1989). Modelos de aprendizaje en la didáctica de las ciencias. Investigación en la Escuela, 9, pp. 17-24.

BELTRÁN, J.A. (1993). Procesos, estrategias y técnicas de aprendizaje. Madrid: Síntesis.

BRINGUIER, J. (1977). Conversaciones con Piaget. Barcelona: Granica.

CAREY, S. (1991). Knowledge acquisition: Enrichment or conceptual change?, en Carey, S. y Gelman, R., The epigenesis of mind. Hillsdale, Nueva Jersey: Erlbaum.

CLAXTON, G. (1984). Vivir y aprender. Madrid: Alianza Editorial.

COLL, C. (1995). Un marco de referencia psicológico para la educación escolar: la concepción constructivista del aprendizaje y de la enseñanza, pp. 435-453, en Coll, C., Palacios, J. y Marchesi, A., Desarrollo psicológico yeducación, II. Psicología de la Educación. Madrid: Alianza Editorial.

DUSCHL, R.A. (1994). Editorial Policy Statement and Introduction. Science Education, 78(3), pp. 203-208.

GARCÍA MADRUGA, J.A. (1995). Aprendizaje por descubrimiento frente a aprendizaje por recepción. La teoría del aprendizaje verbal significativo, pp. 81-93, en Coll, C. Palacios, J. y Marchesi, A., Desarrollo psicológico yeducación, II. Madrid: Alianza Editorial.

GIL, D. (1991). ¿Qué hemos de saber y saber hacer los profesores de ciencias? Enseñanza de las Ciencias, 9(1), pp. 69-77.

GIL, D. (1993). Contribución de la historia y de la filosofía de las ciencias al desarrollo de un modelo de enseñanzaaprendizaje como investigación. Enseñanza de las Ciencias, 11(2), pp. 197-212.
Y bueno sería no olvidar que el cambio conceptual supone, en el mejor de los casos, una pequeña porción de la diversidad de modos que tiene el alumno para adquirir nuevos conocimientos y desarrollar los que ya posee.

Quiero expresar mi agradecimiento a las revisiones sobre la crítica al cambio conceptual hechas por Alicia Benarroch, Javier Perales, José María Posada, José María Oliva y alumnos de doctorado; las apostillas que cada uno realizó sobre las distintas zonas de la línea argumental me permitieron enriquecer sustancialmente el texto final.
GILBERT, J.K. (1995). Studies and fields: directions of Research in Science Education. Studies in Science Education, 25, pp. 173-197.

GUTIÉRREZ, R. (1987). La investigación en didáctica de las Ciencias: elementos para su comprensión. Vol. XXXIX. Madrid: Bordón.

GUTIÉRREZ, R. (1990). La formación del profesorado de ciencias. Valencia: Editorial Nau.

JIMÉNEZ GÓMEZ, E., SOLANO, I. y MARÍN, N. (1997). Evolución de la progresión de la delimitación de las «ideas» de alumno sobre fuerza. Enseñanza de las Ciencias, 15(3), pp. 309-328.

KYLE, W.C. Jr. (1994). Editorial. Journal of Research in Science Teaching, 31(4), pp. 321-322.

LAWSON, A.E. (1983). Predicting Science achievement: the role of development level, disembedding ability, mental capacity, prior knowledge and beliefs. Journal of Research in Science Teaching, 20(2), pp. 117-129.

LUFFIEGO, M., BASTIDA, M.F., RAMOS, F. y SOTO, J. (1994). Systemic model of conceptual evolution. International Journal of Science Education, 16(3), pp. 305-313.

MARTÍNEZ TORREGROSA, J., DOMÈNECH, J.J. y VERDÚ, R. (1993). Del derribo de ideas al levantamiento de puentes: La epistemología de la ciencia como criterio organizador de la enseñanza de las ciencias física y química. Qurriculum, 6 , pp. 67-89.

MARÍN, N. (1984). Evaluación de dos métodos experimentales en la enseñanza de la física básica. (Tesis inédita.) Granada.

MARÍN, N. (1984). Cuaderno de experimentos de física para maestros, pp. 85-92, en Actas de IV Encuentros de Didáctica 


\section{DEBATES}

de Física y Química. Cádiz: Servicio de publicaciones de la Universidad de Cádiz.

MARÍN, N. (1985). Evaluación del aprendizaje por descubrimiento en física, pp. 86-92, en Actas de los V Encuentros de Didáctica de Física y Química. Córdoba: Tipografía Católica.

MARÍN, N. (1986). Estudios sobre las SRT de M. Shayer. VII Encuentros de Didáctica de la Física y Química. Jaén.

MARÍN, N. (1995). Metodología para obtener información del alumno de interés didáctico. Almería: Servicio de Publicaciones de la Universidad de Almería.

MARÍN, N. (1998). Fundamentos de didáctica de las ciencias experimentales. Almería: Servicio de Publicaciones de la Universidad de Almería.

MARÍN, N., SOLANO, I. y JIMÉNEZ GÓMEZ, E. (En revisión.) Tirando del hilo de la madeja constructivista. Enseñanza de las Ciencias.

NOVAK, J.D. y GOWIN, D.B. (1988). Aprendiendo a aprender. Barcelona: Martínez Roca.
OLIVA, J.M. (1996). Estudios sobre la consistencia en las ideas de los alumnos en ciencias. Enseñanza de las Ciencias, 14(1), pp. 87-92.

PIAGET, J. (1976). La toma de conciencia. Madrid: Morata.

PIAGET, J.(1978). La equilibración de las estructuras cognitivas. Problema central del desarrollo. Madrid: Siglo XXI.

POSADA, J.M. (1996). Hacia una teoría sobre las ideas científicas de los alumnos: influencia del contexto. Enseñanza de las Ciencias, 14(3), pp. 303-314.

POZO, J.I. (1989). Teorías cognitivas del aprendizaje. Madrid: Morata.

RUMELHART, D.E. y ORTONY, A. (1982). The representation of knowledge in memory. Infancia y aprendizaje, 20, pp. 115-158.

SHAYER, M. (1979). Science Reasoning Tasks. Windsor: NFER-NELSON.

VUYK, R. (1985). Panorámica y crítica de la epistemología genética de Piaget 1965-1980. Madrid: Alianza Universitaria. 